\title{
Fixed volume particle trace emission for the analysis of left atrial blood flow using 4D Flow MRI
}

Stephen Gaeta, Petter Dyverfeldt, J onatan Eriksson, Carljohan Carlhäll, Tino Ebbers and Ann F Bolger

The self-archived postprint version of this journal article is available at Linköping University Institutional Repository (DiVA):

http:// urn.kb.se/ resolve?urn=urn:nbn:se:liu:diva-147080

N.B.: When citing this work, cite the original publication.

Gaeta, S., Dyverfeldt, P., Eriksson, J ., Carlhäll, C., Ebbers, T., Bolger, A. F, (2018), Fixed volume particle trace emission for the analysis of left atrial blood flow using 4D Flow MRI, Magnetic

Resonance Imaging, 47, 83-88. https:/ / doi.org/ 10.1016/j.mri.2017.12.008

Original publication available at:

https:// doi.org/ 10.1016/j.mri.2017.12.008

Copyright: Elsevier

http:// www.elsevier.com/

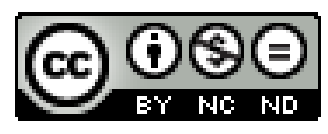


Fixed Volume Particle Trace Emission for the Analysis of Left Atrial Blood Flow using 4D Flow MRI

Stephen Gaeta ${ }^{1 *}$, Petter Dyverfeldt ${ }^{2,3 *}$, Jonatan Eriksson ${ }^{2,3}$, Carl-Johan Carlhäll ${ }^{2-4}$, Tino Ebbers $^{2,3}$, and Ann F. Bolger ${ }^{2,5}$

* Equal contributions

Author Affiliations:

1. Department of Medicine, Duke University, Durham, NC, United States

2. Division of Cardiovascular Medicine, Department of Medical and Health Sciences, Linköping University, Linköping, Sweden

3. Center for Medical Image Science and Visualisation (CMIV), Linköping University, Linköping, Sweden

4. Department of Clinical Physiology, Linköping University, Linköping, Sweden

5. Department of Medicine, University of California San Francisco, San Francisco, CA, United States

Corresponding Author Info: Petter Dyverfeldt, Linköping University Hospital, IMH/KVM, 58183 Linköping, Sweden 


\section{ABSTRACT}

4D Flow MRI has been used to quantify normal and deranged left ventricular blood flow characteristics on the basis of functionally distinct flow components. However, the application of this technique to the atria is challenging due to the presence of continuous inflow. This continuous inflow necessitates plane-based emission of particle traces from the inlet veins, leading to particles that represents different amounts of blood, and related quantification errors. The purpose of this study was to develop a novel fixed-volume approach for particle tracing and employ this method to develop quantitative analysis of 4D blood flow characteristics in the left atrium. 4D Flow MRI data were acquired during free-breathing using a navigator-gated gradientecho sequence in three volunteers at 1.5T. Fixed-volume particle traces emitted from the pulmonary veins were used to visualize left atrial blood flow and to quantitatively separate the flow into two functionally distinct flow components: Direct flow = particle traces that enter and leave the atrium in one heartbeat, Retained flow = particle traces that enter the atrium and remains there for one cardiac cycle. Flow visualization based on fixed-volume traces revealed that, beginning in early ventricular systole, flow enters the atrium and engages with residual blood volume to form a vortex. In early diastole during early ventricular filling, the organized vortical flow is extinguished, followed by formation of a second transient atrial vortex. Finally, in late diastole during atrial contraction, a second acceleration of blood into the ventricle is seen. The direct and retained left atrial flow components were between $44-57 \%$ and $43-56 \%$ of the stroke volume, respectively. In conclusion, fixed-volume particle tracing permits separation of left atrial blood flow into different components based on the transit of blood through the atrium.

Key Words: 4D Flow MRI, MR Flow Imaging, left atrium, blood flow, cardiovascular disease, cardiovascular physiology 


\section{INTRODUCTION}

Blood flow patterns have been postulated to influence the efficiency of pump function through energy preserving redirection of blood through the heart (1) Three-dimensional cine phasecontrast MRI with three-directional velocity encoding (4D Flow MRI) permits detailed investigations of complex cardiovascular blood flow (2). Blood flow can be visualized in a straight-forward and intuitive manner and visualized flow paths can be quantitatively analyzed to reveal information about the kinetic energy, speed, travel distance, etc., of different functional components of blood flow. One way of achieving such information for blood blow in the ventricles of the heart is to emit virtual blood particles backwards and forwards in time from a uniform volumetric grid in the LV or RV at the time of end-diastole and trace these particles to end systole $(3,4)$. In this "volumetric and single time-point emission" approach, each virtual blood particle represents the same amount of blood. Analysis of the resulting pathlines permits quantification of the volume and kinetic energy of distinct sub-components of ventricular blood flow with minimal user interaction (3).

The "volumetric" approach to particle emission used in the ventricles relies on the presence of a time-point during which there is no flux in or out of the ventricle (isovolumetric contraction). Unfortunately, the "volumetric" particle emission approach is insufficient in cardiovascular structures with continuous inflow, such as blood vessels and atria. Blood flow in vessels and atria requires monitoring of particles emitted from planes throughout the cardiac cycle. Such a "plane-based" approach typically emits particles at fixed time-intervals $(5,6)$. As the volume of blood represented by each particle equals the area of the grid vertex multiplied by the product between the velocity of blood and the emission time-interval, "plane-based" emission results in particles that represent different amounts of blood (see Figure 1A). When the volume of each trace is taken into account, plane-based pathlines permits quantitative analysis of parameters such as kinetic energy, as previously demonstrated for the left ventricle (5). Nevertheless, variable particle volumes will lead to quantification errors when standard integration techniques are employed, as these typically integrate particle positions with the simplifying assumption of massless or uniform mass particles. This is a valid approach in the case of volumetric particle emission, but is incorrect when fixed time-step plane-based particle emission is used. Additionally, even if the variable volume of particles is accounted for in a quantitative analysis, 
varying particle volume makes visual interpretation of pathlines visualizations less straightforward as these visualizations are normally performed with stereotyped particle representations.

To overcome these important limitations in the current definition of plane-based particle traces, we propose variable time-step, or "flux-based", particle emission (see Figure 1B) and will explore if this approach permits quantitative analysis of atrial blood flow. To that end, we sought to confirm the ability of a plane-based emission strategy to identify the left atrial blood volume as well as to develop an analysis method by which distinct subsets of left atrial flow can be quantified.

\section{MATERIALS AND METHODS}

\subsection{MRI Data}

MRI data were acquired in three healthy volunteers (19, 21, 22 years old; 2 female) using a $1.5 \mathrm{~T}$ Philips Achieva scanner (Philips Healthcare, Best, the Netherlands). The study was approved by the regional Ethical Review Board and all subjects gave written informed consent before participation.

4D Flow MRI data were acquired during free-breathing using a navigator-gated gradient-echo sequence with the following parameters: VENC $=100 \mathrm{~cm} / \mathrm{s}$, flip angle $=8^{\circ}$, echo time $=3.7 \mathrm{~ms}$, repetition time $=6.3 \mathrm{~ms}$, parallel imaging $($ SENSE) speed-up factor $=2$, $\mathrm{k}$-space segmentation factor $=2,6 \mathrm{~mm}$ navigator window size. The spatial resolution was $3 \times 3 \times 3 \mathrm{~mm}^{3}$ and the field-

of-view (FOV) was adjusted for each subject to cover the left heart with a double-oblique slab orientation. Scan time was about 10-15 minutes excluding the navigator efficiency, which was around 50\%. Following the 3DcinePC-MRI acquisition, the flow data was reconstructed into 40 time frames. Corrections were made for concomitant gradient fields, phase-wraps, and background phase errors $(7,8)$. 
Morphological 2-, 3- and 4-chamber long-axis and the stack of short-axis images were acquired using cine balanced steady-state free-precession (bSSFP). The images were acquired in 30 time frames during end-expiratory breath holds. Slice thickness was $8 \mathrm{~mm}$. Acquired and reconstructed pixel size for the short-axis images was $2.19 \times 1.78 \mathrm{~mm}^{2}$ and $1.37 \times 1.37 \mathrm{~mm}^{2}$, respectively.

Two-dimensional cine through-plane PC-CMR velocity data were acquired in a plane perpendicular to the ascending aorta just downstream from the aortic valve. Imaging parameters included velocity encoding range $(\mathrm{VENC})=200 \mathrm{~cm} / \mathrm{s}$, echo time $(\mathrm{TE})=3 \mathrm{~ms}$, repetition time $(\mathrm{TR})=5 \mathrm{~ms}$, flip angle $=15^{\circ}$, slice thickness $=7 \mathrm{~mm}$, and pixel size $=1.6 \times 1.6 \mathrm{~mm}^{2}$. Three lines of k-space were acquired per heartbeat, resulting in a temporal resolution of $30 \mathrm{~ms}$.

\subsection{Segmentation of Left Atrium}

Time-resolved left atrial anatomy was defined in the short-axis bSSFP images using freely available segmentation software (Segment, version 1.9). Briefly, the endocardial border of shortaxis slices was identified by visual inspection and defined at each time step for all slices containing the left atrium (6-9 slices at end-systole for the patients included in this study). The border was confined to the body of the atrium, without extension into the pulmonary veins. The most apical slice (which varied throughout the cardiac cycle) was defined as the mitral annulus plane for later analysis. After segmentation, the left atrial volume was interpolated to 4 millimeter slices by proximal interpolation. Left atrial volume was computed at each time point.

\subsection{Particle Emission and Pathlines}

Square planes with a 10x10 grid were placed orthogonally across each of the pulmonary veins near their attachment to the LA using visualization software Ensight (CEI Inc., Apex, NC, USA). Planes were between 2 and $2.5 \mathrm{~cm}$ on each side, with all planes identical for a given subject. Positioning was guided by visual inspection of morphologic images and backwards streamlines from the left atrium which indicated sources of inflow. 2 of 3 patients studied had 4 pulmonary veins; 1 patient had 2 right pulmonary veins and a single left pulmonary vein (that bifurcated proximal to its insertion) and therefore only had 3 pulmonary vein planes placed. The lumen of the pulmonary veins was delineated in Matlab (The MathWorks Inc., Natick, MA, USA). Virtual 
blood particles were emitted from these delineated 2D planes using both fixed time-step particle emission and flux-based particle emission.

Conventional fixed time-step particle emission was done by emitting particles at times separated by a constant time step $d t$ (see Figure 1A). Particles were emitted throughout one cardiac cycle, starting at onset systole, and were traced until end diastole. Particles were not emitted when flow was directed out of the atrium.

The proposed "flux-based" particle emission (see Figure 1B) emits particles that represents a uniform blood volume ("particle volume"). By changing the time step of particle emission dynamically throughout the cardiac cycle dependent on local instantaneous blood flow velocity, a pre-determined/normalized volume of blood is allowed to pass between each particle's creation. Specifically, for each pixel in an emitter plane, the time step $(d t)$ at time $t$ is derived by the flux equation as

$$
d t=\frac{V}{A|\vec{v}(t)| \cos \theta}
$$

where $V$ is the pre-determined volume of blood to be represented by each particle, $A$ is the area of each pixel, and $\vec{v}$ is the instantaneous flow velocity at an angle $\theta$ to the normal vector of the plane. In this study, excessively long $d t$ due to low-velocity flow was avoided by ignoring velocities $V$ causing dt $>20$. When such low velocity was present, determination of dt and particle creation were paused until $\mathrm{dt}<20$, which was checked at $1 \mathrm{~ms}$ intervals.. Particles were emitted throughout one cardiac cycle, starting at onset systole, and were traced until end diastole. Particles were not emitted when flow was directed out of the atrium.

The particles were forward integrated to create pathlines by $4^{\text {th }}$ order Runge-Kutta numerical integration with $5 \mathrm{~ms}$ time step using in-house Matlab scripts. The location and time history of each pathline was recorded for subsequent automated analysis. To confirm that the plane-based emission strategy captures the entire blood volume of interest, the blood volumes captured by 
fixed time-step and flux-based emission were compared to the stroke volume calculated by integration of the flow curve in the ascending aorta 2D flow data.

\subsection{Flow component analysis}

By comparing the position-history of each pathline with the time-resolved segmented left atrial volume to determine whether they were inside or outside the chamber, the pathlines were categorized into the following functional flow components:

- $\quad$ Direct flow = Pathlines that entered the left atrium, crossed the mitral annulus (defined as the most apical slice of the segmented volume) and resided outside of the left atrial volume at the end of the analyzed cardiac cycle.

- Retained inflow = Pathlines that entered the left atrium and did not subsequently leave through the same pulmonary vein or cross the mitral annulus within the analyzed cardiac cycle.

- "Reversed flow" = Pathlines that entered the left atrium but exited through the same PV plane.

The volumes of blood already residing in the atrium at the beginning of the cardiac cycle that enters the ventricle, so-called "delayed ejection flow" and "residual volume", are not visualized by this method, but their volumes can be derived by exploiting that delayed ejection flow volume should be equal to the retained inflow volume following the law of conservation of mass and by subtracting the total in/outflow from the LA volume, respectively. Particles that were emitted from the square-shaped emitter planes but never entered the segmented left atrial volume were considered as "junk" and excluded from further analysis.

\subsection{Kinetic energy calculation}

The kinetic energy was calculated for each pathline at time $t$ by its speed $v$, as $K E=\frac{1}{2} \rho V v(t)^{2}$,

where particle mass was determined by its volume $V$ and the density of blood $\rho$ taken as 1060 $\mathrm{kg} / \mathrm{m}^{3}$. To discriminate the contribution of velocity and volume to each pathline's kinetic energy, "kinetic energy density" was calculated by scaling the kinetic by the volume of blood represented by each pathline, as in Refs. $(9,10)$. 


\section{RESULTS}

The plane-based emission strategy was confirmed to capture the entire blood volume of interest; the net PV flow was slightly underestimated when compared to the aorta stroke volume for both fixed time-step and flux-based emission (see Table 1).

Flow component results for a representative subject are shown in Figure 2, with flow separated into direct (green) and retained (yellow) flow components. Beginning in early ventricular systole (a), flow enters the atrium and engages with residual blood volume (not visualized) to form a vortex (b). In early diastole during early ventricular filling (c), the organized vortical flow is extinguished, followed by formation of a second transient atrial vortex (d). Finally, in late diastole during atrial contraction, a second acceleration of blood into the ventricle is seen (e). In this subject, the proportion of direct flow and retained inflow was $57.1 \%$ and $42.9 \%$, respectively.

The impact of different plane-based emission strategies on flow component analysis is shown in Figure 3. Although the volume of separate flow components are similar when either fixed time step or flux-based emission is used (Figure 3A), relative under-sampling of high-velocity flow by fixed time step analysis confers a large difference in the proportion of pathlines that represent each component (Figure 7B,C). For this subject, fixed time step analysis under-samples direct flow (which is higher velocity) and over-samples the low velocity, reversed flow. Additionally, fixed time step emission creates a higher proportion of particles that are emitted from the outer parts of the emitter plane and never enter the LA (junk).

The volume, kinetic energy, and kinetic energy density of the separated flow components from this same patient are shown in Figure 4. For this patient, direct flow enters the atrium earlier in the cardiac cycle, primarily during ventricular systole. Diastolic atrial inflow is predominantly retained flow and flow reversal into the pulmonary veins is seen in late diastole. Three peaks in the total kinetic energy are seen. The relative contributions of acceleration and accumulation of blood volume to the kinetic energy can be inferred by comparing kinetic energy (Figure 4B) with the total volume (Figure 4A) and kinetic energy density (Figure 5C). For this patient, the first 
peak in kinetic energy (during ventricular systole) is due primarily to the kinetic energy of direct flow volume. The kinetic energy density of direct and retained flow is equivalent during this time, however, indicating that the difference is due to a larger volume of direct flow (as seen in Figure 5A) rather than a difference in the relative velocity of these flow components. The largest increase in $\mathrm{KE}$ is seen in the direct flow component during early diastole. As evidenced by its higher kinetic energy density, this is primarily due to a large increase in its velocity at this time (due to acceleration into the left ventricle). A final, smaller peak in kinetic energy is seen in late diastole, corresponding to a modest increase in the velocity of direct, retained, and reversed flow, coinciding with atrial contraction.

\section{DISCUSSION}

This paper presented a method for semi-automated quantification and visualization of distinct functional components of LA blood flow. The method separates LA blood flow into different functional components depending on their path through the LA. Parameters such as flow volumes, velocity, and kinetic energy are quantified for each component throughout the cardiac cycle. This method was achieved by introducing of a novel flux-based approach for particle trace emission that corrects the non-uniformity in particle volume and implicit oversampling of low velocity flow that occurs with existing flow visualization techniques. This approach was shown technically feasible, accurate, and to avoid inaccuracies inherent to existing methods of particle emission.

Identification of the separate functional components of left atrial blood flow with 4D Flow MRI analysis offers multiple advantages over existing methods for characterization of LA flow dynamics. Prior studies have used time resolved left atrial volume to infer functional components of left atrial blood flow $(11,12)$. One conceptual framework, for example, separates left atrial function into a reservoir, conduit, and stroke volume, with the assumption that changes in their relative volumes can be useful indices for cardiovascular function. The total left atrial volume used to infer these components, however, is itself an epiphenomenon reflecting the overall balance between atrial inflow and outflow (12). An alternative approach to derive these functional volumes, in which MR and echocardiography are combined in the same subject (11), suffers from temporal dissociation of measurements between and within these modalities in 
addition to limited visualization of PVs by echocardiography. Analysis based on 4D Flow MRI offers advantages over these prior strategies by allowing direct visualization of the flow of relevant functional blood flow components.

4D Flow MRI has previously been used to characterize the configuration of normal LA blood flow including the extent and timing of vortices (13), degrees of kinetic energy of LA blood flow, as well as to assess LA mean and median velocity in normal volunteers and atrial fibrillation patients $(14,15)$. Recently, Callaghan et al explored a pathlines-based analysis method similar to the one in the present study (16). Their approach is based on fixed time step emission of pathlines which are traced over not one but two cardiac cycles and take into account only those particles that exit the RA during the subsequent heartbeat and a definition of RA volume which is based not on the anatomical volume of the RA but on the portion of the RA that is traversed by particle traces that enter the RA from the caval veins during one cardiac cycle and exit to the RV during the subsequent heartbeat (16). Consequently, although using the same terminology as in the present study, their flow components have a somewhat different meaning than the ones reported here. Of note, the definition of flow components used in the present study follows that previously used for the $\operatorname{LV}$ and $\mathrm{RV}(3,4)$. Although the nature of ventricular versus atrial blood flow requires different approaches to capture these flow components, we believe that a uniform flow component definition is helpful for comparisons between cardiac chambers.

The standard approach of fixed time step particle emission can introduce errors through implicit variability in the blood volume represented by each particle as well as oversampling of low velocity flow. This is problematic because integration of particle positions to create pathlines is normally done with the simplifying assumption of uniform particle mass. Similarly, while total inflow volume/kinetic energy calculations will nevertheless be accurate with fixed time step particle emission when the relative contribution of each particle to the total is scaled by its individual volume/mass, the relative volumes of the separate flow components, which are dependent on integrated pathlines, can be inaccurate. Even the standard visualization technique, in which pathlines are represented by particles of uniform size, incorrectly implies that each pathline represents the same amount of blood volume. Flux-based emission, in which the blood volume of each particle is indeed the same, is necessary to correct these inaccuracies. We 
speculate that the errors associated with fixed time step emission will be more pronounced in contexts with more variation in flow velocity and, therefore, in particle volumes such as in the great vessels. This may be further exacerbated in locations with less complete mixing of blood volumes and more functionally distinct flow components, such as vessel bifurcations.

This study has several limitations. The study was methodologically oriented and only included three young subjects and therefore conclusions regarding normal values of LA flow components cannot be drawn. However, although application of this techniques to larger numbers of subjects is necessary before generalizations can be made, the total calculated $\mathrm{KE}$ is comparable to that seen in prior studies (10).

In conclusion, fixed volume particle emission permits sampling of LA blood volumes and intuitive visualizations where each trace represents the same volume of blood. Using fixedvolume particle traces, LA flow can be separated into different components based on the transit of blood through the LA. Quantitative analysis of functionally distinct subsets of LA flow may provide new perspectives on LA function in health and disease.

\section{REFERENCES}

1. Kilner PJ, Yang GZ, Wilkes AJ, Mohiaddin RH, Firmin DN, Yacoub MH: Asymmetric redirection of flow through the heart. Nature 2000; 404:759-761.

2. Dyverfeldt P, Bissell M, Barker AJ, et al.: 4D flow cardiovascular magnetic resonance consensus statement. J Cardiovasc Magn Reson 2015; 17.

3. Eriksson J, Carlh?ll C, Dyverfeldt P, Engvall J, Bolger A, Ebbers T: Semi-automatic quantification of 4D left ventricular blood flow. J Cardiovasc Magn Reson 2010; 12.

4. Fredriksson AG, Zajac J, Eriksson J, et al.: 4-D blood flow in the human right ventricle. Am J Physiol - Hear Circ Physiol 2011; 301.

5. Bolger AF, Heiberg E, Karlsson M, et al.: Transit of blood flow through the human left ventricle mapped by cardiovascular magnetic resonance. J Cardiovasc Magn Reson 2007; 9:741-747.

6. Bachler P, Valverde I, Uribe S: Quantification of caval flow contribution to the lungs in vivo after total cavopulmonary connection with 4-dimensional flow magnetic resonance imaging. $J$ 
Thorac Cardiovasc Surg 2012; 143:742-743.

7. Wigstrom L, Ebbers T, Fyrenius A, et al.: Particle trace visualization of intracardiac flow using time-resolved 3D phase contrast MRI. Magn Reson Med 1999; 41:793-799.

8. Ebbers T, Haraldsson H, Dyverfeldt P, Sigfridsson A, Warntjes M, Wigström L: Higher order weighted least-squares phase offset correction for improved accuracy in phase-contrast MRI. In Proc 15th Int'l Soc Magn Reson Med. Berlin, Germany; 2007:1367.

9. Carlsson M, Heiberg E, Toger J, Arheden H: Quantification of left and right ventricular kinetic energy using four-dimensional intracardiac magnetic resonance imaging flow measurements. Am J Physiol Circ Physiol 2012; 302:H893-H900.

10. Arvidsson P, Toger J, Heiberg E, Carlsson M, Arheden H: Quantification of left and right atrial kinetic energy using four-dimensional intracardiac magnetic resonance imaging flow measurements. J Cardiovasc Magn Reson 2013; 15:P218.

11. Bowman AW, Kovács SJ: Left atrial conduit volume is generated by deviation from the constant-volume state of the left heart: a combined MRI-echocardiographic study. Am J Physiol Circ Physiol 2004; 286:H2416-H2424.

12. Germans T, Götte MJW, Nijveldt R, et al.: Effects of aging on left atrioventricular coupling and left ventricular filling assessed using cardiac magnetic resonance imaging in healthy subjects. Am J Cardiol 2007; 100:122-127.

13. Fyrenius A, Wigström L, Ebbers T, Karlsson M, Engvall J, Bolger AF: Three dimensional flow in the human left atrium. Heart 2001; 86:448-455.

14. Fluckiger JU, Goldberger JJ, Lee DC, et al.: Left atrial flow velocity distribution and flow coherence using four-dimensional FLOW MRI: A pilot study investigating the impact of age and Pre-and Postintervention atrial fibrillation on atrial hemodynamics. J Magn Reson Imaging 2013; 38:580-587.

15. Markl M, Carr M, Ng J, et al.: Assessment of left and right atrial 3D hemodynamics in patients with atrial fibrillation: a 4D flow MRI study. Int J Cardiovasc Imaging 2016; 32:807815.

16. Callaghan FM, Arnott C, Figtree GA, Kutty S, Celermajer DS, Grieve SM: Quantifying right atrial filling and emptying: A 4D-flow MRI study. J Magn Reson Imaging 2017; 45:1046-1054. 


\section{FIGURES}
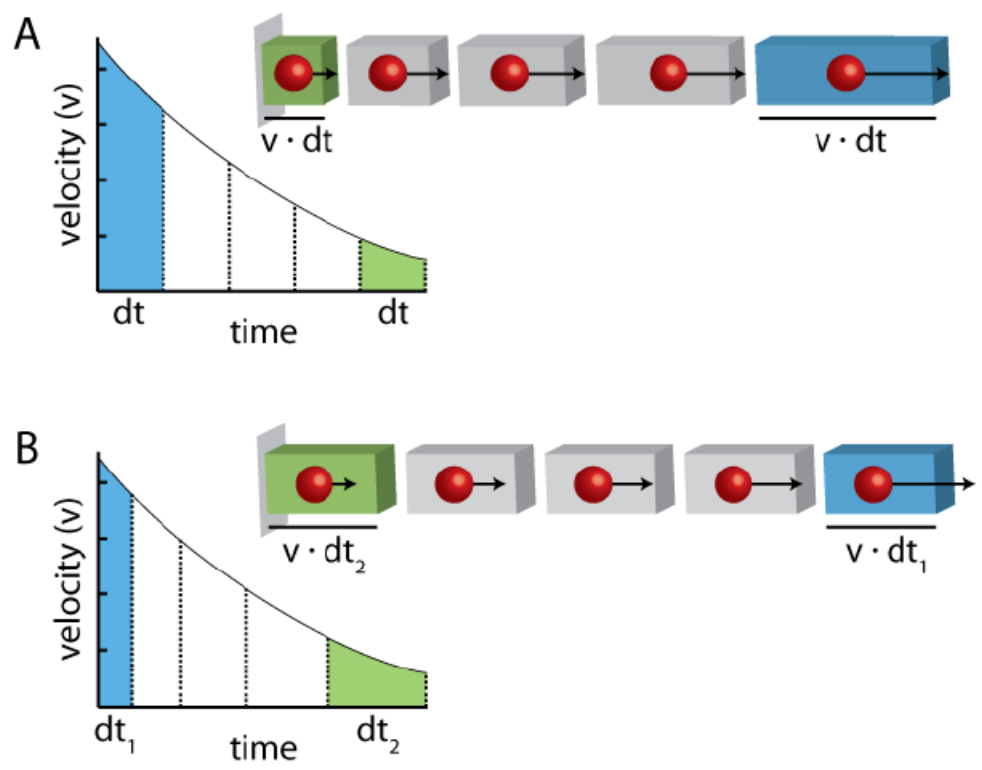

Figure 1. Two different approached to plane-based particle emission. A: The traditional particle emission technique emits particles (red spheres) from each point on a plane at fixed time intervals (dt). By doing so, particles emitted during high speed flow (blue) will represent a larger blood volume than those emitted during low speed flow (green), leading to inaccurate depictions and measurements of actual volume flux. B: By changing the time step dynamically, with shorter time steps during high speed flow (blue) and longer time steps during low speed flow (green), the blood volume represented by each particle is normalized, improving the quantitative information of visualization and accuracy of volume estimates. 

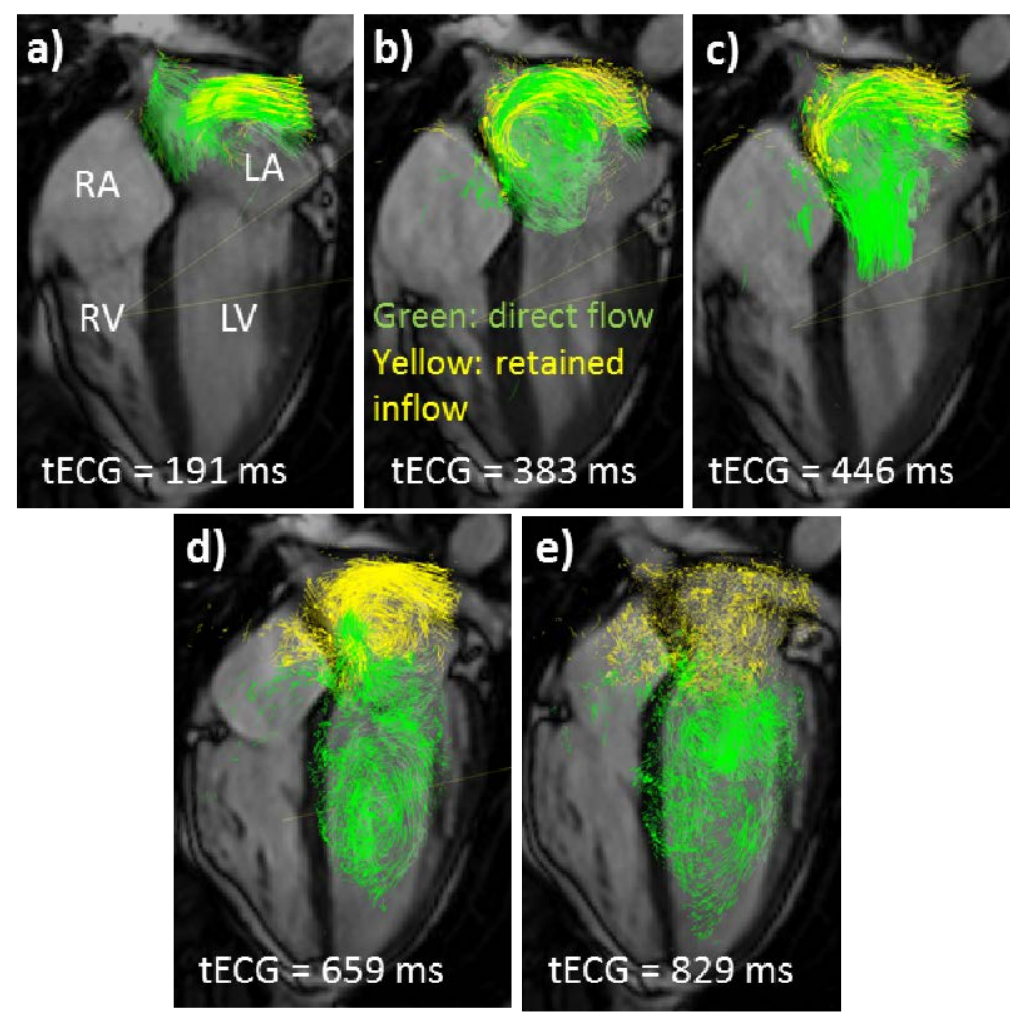

Figure 2. Visualization of LA flow for one representative volunteer, with flow separated into direct (green) and retained (yellow) flow components. Beginning in early ventricular systole (a), flow enters the atrium and engages with residual blood volume (not visualized) to form a vortex (b). In early diastole during early ventricular filling (c), the organized vortical flow is extinguished, followed by formation of a second transient atrial vortex (d). Finally, in late diastole during atrial contraction, a second acceleration of blood into the ventricle is seen (e). 


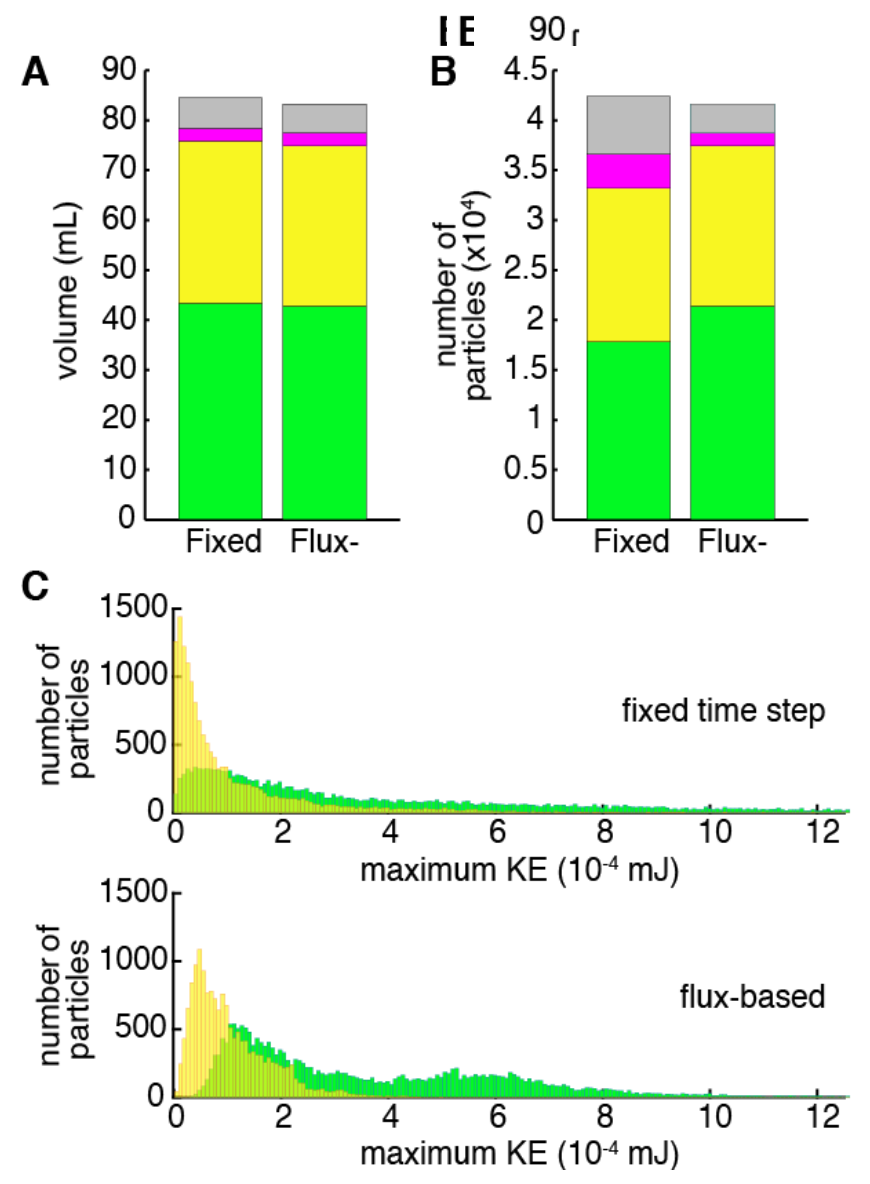

Figure 3. A: Differences in sampling of flow components between fixed time step and flux-based analysis. The proportions of direct flow (green), retained flow (yellow), reversed flow (magenta), and extraneous volume (gray) are shown, demonstrating the relative undersampling of direct flow and oversampling of reversed and extraneous flow by the fixed time step method compared to flux-based emission. B: Relative volumes of flow components calculated by fixed time step and flux-based methods. Data analysis performed on the same representative healthy subject as in prior figures, comparing fixed time step of $20 \mathrm{~ms}$ to flux-based emission with particle volume $0.01 \mathrm{~mL}$, such that each method leads to a similar number of particles created. C: distribution of time steps used in flux-based particle emission demonstrates oversampling of low velocity flow (low maximum KE) with the fixed time step method. 

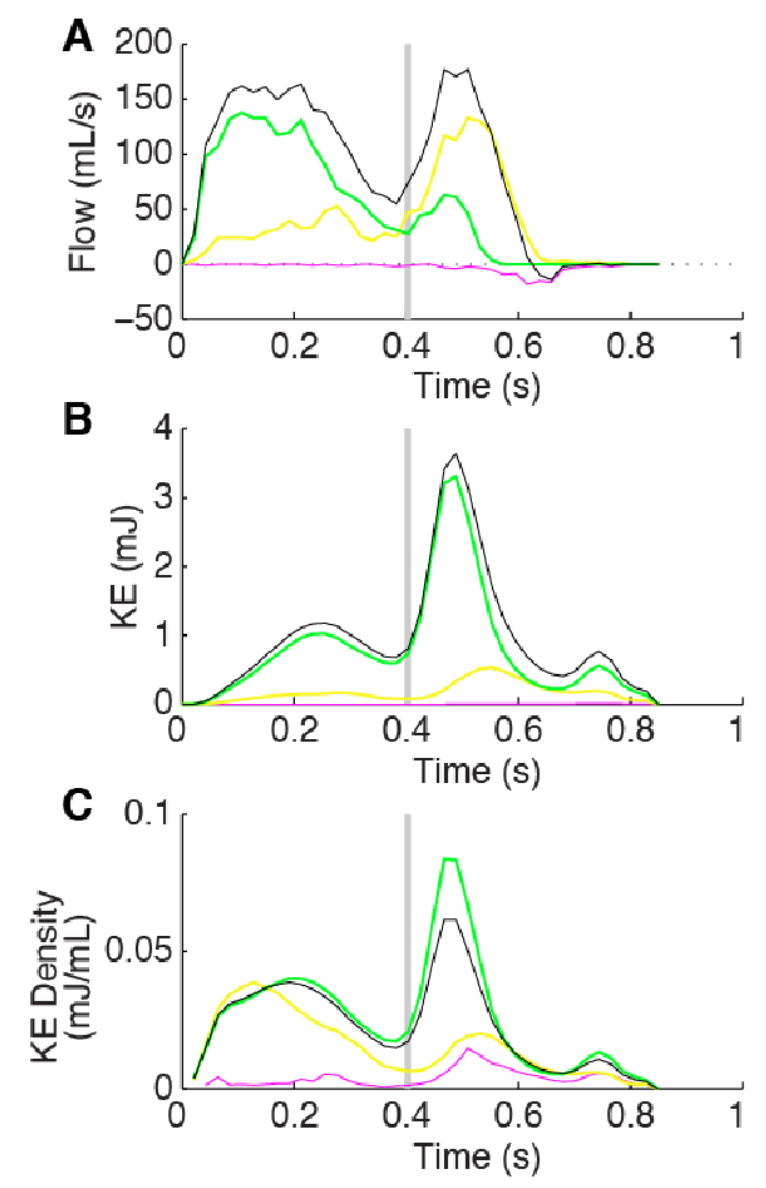

Figure 4. Quantification of left atrial flow parameters in a representative healthy subject over one cardiac cycle (beginning from ventricular systole; end-systole indicated with vertical gray line) including inflow rate (A), kinetic energy (B), and kinetic energy density (C). Flow is separated into total left atrial flow (summed from all four pulmonary veins; black), direct flow (green), retained flow (yellow), and reversed flow (magenta). Data analysis performed with fluxbased particle emission; particle volume of $0.01 \mathrm{~mL}$. 


\section{TABLES}

Table 1. 4D flow results for three healthy subjects using fixed time step and flux-based analysis. Through-plane measurement of stroke volume from the ascending aorta is included for comparison.

\begin{tabular}{|c|c|c|c|c|c|c|c|c|c|c|c|c|c|}
\hline \multirow[b]{2}{*}{ Subject } & \multicolumn{2}{|c|}{ Parameters } & \multicolumn{2}{|c|}{ Number of Particles } & \multicolumn{2}{|c|}{ Net Inflow (mL) } & \multicolumn{2}{|c|}{$\begin{array}{c}\text { Direct Flow (\%Net } \\
\text { Inflow) }\end{array}$} & \multicolumn{2}{|c|}{$\begin{array}{c}\text { Retained Inflow } \\
\text { (\%Net Inflow) }\end{array}$} & \multicolumn{2}{|c|}{ Reversed Flow (mL) } & \multirow[t]{2}{*}{$\begin{array}{c}\text { Aorta } \\
\text { Stroke } \\
\text { Volume }\end{array}$} \\
\hline & $\begin{array}{c}\text { Fixed time- } \\
\text { step } \\
\text { dt (ms) }\end{array}$ & $\begin{array}{c}\text { Flux- } \\
\text { based } \\
\text { Particle } \\
\text { vol. (mL) }\end{array}$ & $\begin{array}{l}\text { Fixed } \\
\text { TS }\end{array}$ & $\begin{array}{l}\text { Flux- } \\
\text { Based }\end{array}$ & $\begin{array}{c}\text { Fixed } \\
\text { TS }\end{array}$ & $\begin{array}{l}\text { Flux- } \\
\text { Based }\end{array}$ & $\begin{array}{l}\text { Fixed } \\
\text { TS }\end{array}$ & $\begin{array}{l}\text { Flux- } \\
\text { Based }\end{array}$ & $\begin{array}{l}\text { Fixed } \\
\text { TS }\end{array}$ & $\begin{array}{l}\text { Flux- } \\
\text { Based }\end{array}$ & $\begin{array}{c}\text { Fixed } \\
\text { TS }\end{array}$ & $\begin{array}{l}\text { Flux- } \\
\text { Based }\end{array}$ & \\
\hline 1 & 14 & 0.010 & 10892 & 11150 & 102.64 & 100.20 & 44.00 & 44.25 & 56.00 & 55.75 & 2.95 & 2.61 & 108.31 \\
\hline 2 & 20 & 0.010 & 7478 & 8219 & 76.09 & 74.42 & 57.04 & 57.09 & 42.96 & 42.91 & 2.45 & 2.27 & 82.44 \\
\hline 3 & 10 & 0.010 & 7820 & 7698 & 69.00 & 67.78 & 53.70 & 53.94 & 46.30 & 46.06 & 0.41 & 0.33 & 80.29 \\
\hline
\end{tabular}

